Meta

Journal des traducteurs

Translators' Journal

\title{
Pas de deux : le mésocosme de la traduction comme matrice d'une sémantique frontalière
}

\section{Laurent Lamy}

Volume 40, numéro 3, septembre 1995

La traduction, qu'est-ce à dire? Phénoménologies de la traduction

URI : https://id.erudit.org/iderudit/003390ar

DOI : https://doi.org/10.7202/003390ar

Aller au sommaire du numéro

Éditeur(s)

Les Presses de l'Université de Montréal

ISSN

0026-0452 (imprimé)

1492-1421 (numérique)

Découvrir la revue

Citer cet article

Lamy, L. (1995). Pas de deux : le mésocosme de la traduction comme matrice d'une sémantique frontalière. Meta, 40(3), 461-477.

https://doi.org/10.7202/003390ar
Résumé de l'article

This paper deals with the idea that translation constitutes a reality sui generis, functioning as a manifold matrix sustaining a relation whose openness can not be mastered to form a closed system. Drawing from the core intuition of romantic Kunstsprache, the argument considers the paradoxical notion of nine Sprache put forth by Walter Benjamin, suggesting that its unsolved implications break towards a critical resistance to any semantic-saturation of the contact between tongues formed by diasporic process: the debt of the translator is an involvement towards a "survival" of this plural disclosure. 


\title{
PAS DE DEUX : LE MÉSOCOSME DE LA TRADUCTION COMME MATRICE D'UNE SEMANTIQUE FRONTALIËRE*
}

\author{
LAURENT LAMY \\ Universite de Montréal. Montréal. Canada
}

\begin{abstract}
This paper deals with the idea that translation constitutes a reality sui generis, functioning as a manifold matrix sustaining a relation whose openness can not be mastered to form a closed system. Drawing from the core intuition of romantic Kunstsprache, the argument considers the paradoxical notion of reine Sprache put forth by Walter Benjamin, sug. gesting that its unsolved implications break towards a crifical resistance to any semantic saturation of the contact berween tongues formed by diasporic process: the debt of the translator is an involvement towards a "survival" of this plural disclosure.
\end{abstract}

Il nous a été loisible d'établir de façon à peu près définitive que la traduction littéraire. à l'instar de toute pratique d'écriture, n'est pas un exercice prévisible. objectif et répétable, mais une aventure dans les variations : non pas une science dont les données sont réputées valides sur la base de leur réitérabilité, mais un art des différences. Qui plus est, il devrait étre clair que le traducteur, en tant que récepteur et créateur d'art, est libre d'établir et d'offusquer les paramètres de la traduisibilité. (Willis Bamstone. The Poetics of Translation: ma (rad.)

La distinction ici opérée par W. Barnstonel et la position qu'il assume conséquemment dessinent le partage de deux univers sémantiques commandant des approches nettement différenciées de l'économie du sens et de sa condition générique. Approches que je qualifierai. d'une part. de logico-grammaticale, avec une connotation réductionniste, et de l'autre, de crypto-herméneutique, en ce qu'elle accuse et approfondit la résistance marquée par la gestation plurale des ressources idiomatiques mobilisées par divers «bouillons de culture” dont la cristallisation est relativement perméable et se prête volontiers à divers métissages et processus d'acculturation. Je songe ici tout particulièrement à la plasticité remarquable de la culture yiddish. En gros, on retrouve la bipartition de postulats extrêmement tranchés dans la saisie du "fait» de langage, que George Steiner a fixés dans son ouvrage séminal After Bahel sous les labels respectifs d'universaliste et de monadiste ${ }^{2}$. D'un côté. nous aurions une visée holistique ou cybernétique de la construction du sens dans les langues dites «naturelles», que l'on associe alors à la gestion d'un tuteur transcendantal ou de quelque métalangage qui viendrait coiffer toute disparité en matière d'expression linguistique; de l'autre, on est à pressentir quelque chose comme le frayage diasporique ou disséminal de traces et d'éléments sémiques répondant à une territorialisation (mapping) discrète de l'expérience qui trouve alors son expression la plus aboutie dans le «Dictamen" poétique, pièce de "résistance» sur laquelle vient buter le travail de traduction, qui y trouve certes sa crux mais peut-être aussi sa rédemption comme discipline autonome, bref comme "art des différences" au sens où l'entend W. Barnstone.

Mais on $n$ 'ira pas loin avec une schématisation aussi abstraite. L'approche logicogrammaticale est déjà grosse d'une tradition de pensée qu'on peut retracer, mais très sommairement ici, aux grammaires spéculatives élaborées par les théo-logiciens médiévaux?. dont l'esprit a été pour ainsi dire réactualisé dans le projet de rationalisation que Noam Chomsky a rangé de façon un peu abusive sous l'étiquette de “linguistique cartésienne" 
et qui correspond en l'occurrence à la publication en 1660 de la Grammaire générale et raisomnée d'Antoine Arnauld et de Claude Lancelot', et, en 1662, de La logique ou l'art de penser. du même Arnauld et de Pierre Nicole's. N'ayant pas lieu ici d'examiner par le détail cette tendance plutôt dominante de l'analyse linguistique. je m'en tiendrai donc à ce passage éloquent du Cartesian Linguistics de Noam Chomsky :

La structure profonde, qui exprime le sens, est [...] commune à toutes les langues, car elle n'est que le reflet des formes de la pensée $|\ldots|$ C'est la structure profonde, sous-jacente à l'énoncé effectif, purement mentale, qui véhicule le contenu sémantique de la phrase. Néanmoins, cette structure profonde est liée aux phrases réelles en ceci que chacune des propositions abstraites qui la composent $|. .$.$| pourrait être directement réalisée sous la forme$ d'un jugement composé d'une seule proposition'.

À peu de choses près, mutatis mutandis, nous pouvons ici pressentir une variante de la profession de foi logiciste selon laquelle toute expression douée de sens et formant une idée complète est réductible à la forme d'un jugement prédicatif répondant à une sémantique véri-conditionnelle (truth-conditional semantics). Mais c'est là aussi une façon d'enchâsser la générativité sémantique dans l'analycité syntaxique, en présumant d'une organisation nucléaire universalisable qui serait prégnante dans l'exercice de toute langue donnée. constituant en quelque sorte le capital foncier, sinon le levier ou la pierre angulaire, de l'acte de traduction. Du moins, si on en résume l'apanage à la translatio entre une langue-source et une langue-cible, en escomptant un minimum d'entropie, sinon une faillite de bon aloi dans le transfert d'une poétique à l'autre, au profit d'une adéquation (putative ou virtuelle) dans le contenu véhiculé par la forme qui en appelle à la validation de la référence. À la limite. on pourrait y aller de ce raisonnement bâtard : si la structure profonde correspond à ce qui est susceptible d'être partagé par n'importe quel locuteur de n'importe quelle langue donnée, donc si cette structure profonde exprime le sens et que c"est le sens qu'il faut traduire lorsqu'il y a matière à traduction, alors le traducteur ne traduit rien. Sauf à trafiquer les idiomes dans un espace de résolution frontalier où il $s$ 'agit de remonter la filière des règles transformationnelles permettant de recouvrer la structure profonde à partir de la structure de surface, qui est amovible et facultative.

La relativité des idiomes n'a rien de volatil ou de simplement spectral : il n'y a pas de genre dont les langues seraient les espèces, et les individus-locuteurs des sujets candidats à la "relève" du concept agréant la participation au règne de l'universalité. Dans l'histoire des idées linguistiques, la notion de "relativisme linguistique» est couramment rattachée à l'hypothèse Sapir-Whorf et à ses ascendants allemands, en l'occurrence les importantes considérations sur l'origine. l'essence et l'exercice du langage qui furent développées, de Johann Gottfried Herder à Wilheim von Humboldt, empruntant le relais peu banal du vaste laboratoire mis en chantier par la Bildung romantique?

Comme l'a fort bien démontré Antoine Berman dans son maître-ouvrage $L$ 'épreuve de l'étranger. la Bildung romantique se présentait dans son rapport au langage comme une synthèse des mouvements d'infinitisation et de finitisation d'une sensibilité ainsi habilitée à se saisir du pluralisme endogène de la signification qui précisément ne peut se révéler comme telle qu'à la faveur de cette potentialisation (Potenzierung) et de cette amplification (Erneiterung) impliquées dans la gestation (Bildung) de la forme poétique où se cristallise une subjectivité plurale remise à sa finitude fondamentale comme actualisation de l'infini en germe dans la matrice de l'ouvre, dans les ressorts du poétique (Dichtung). Si l'enjeu et la portée de la traduction deviennent partie prenante du noyau de questionnement dégagé par cette vaste spéculation sur les ressorts du poétique, c'est que le caractère sui-référentiel de la Sprachlehre romantique entraine, voire se donne pour postulat implicite, la destruction de la structure référentielle naturelle (avec les nuances 
qui s'imposent) du langage. L'exercice d'une langue ne peut désormais être relégué à la seule vertu de véhicule ou d'instrument extérieur à la conscience qui s'y saisit, tant dans sa finitude que dans l'infinité des formes du langage qui révèle alors «une essence qui se forme elle-même" (sich selbst bildendes Wesen). L'idée même de langue naturelle, d'une Natursprache se voit ici frappée de caducité, au profit de l'élaboration d'une Kunsisprache. Il en résulte notamment un effet d' "estrangement", ce contact soudain, on ne peut plus intempestif, avec le non-familier dans une contrée où pourtant le commerce le plus fréquent avec les ressources idiomatiques balisant notre participation à une communauté de destin élective ou contingente semblait jusque lors conforter notre assise dans la langue qui nous est censément acquise. C'est cet effet d'Unheimlichkeit, d' «inquiétante étrangeté» qu'évoque Maurice Blanchot dans La part du feu en marquant ce jeu de dissimilation constitutif de la littérature comme inductrice d'extraterritorialité en regard du vecteur monologique corrélé au champ de l'ostension primé par la sémantique vériconditionnelle. Il appert en effet, écrit Blanchot.

... que ce nouveau langage devrait être par rapport à la langue courante ce qu'un texte a traduire est pour le langage qui le traduit : un ensemble de mots ou d'événements que nous comprenons ê saisissons sans doute, à merveille, mais qui, dans leur familiarité même, nous donnent le sentiment de notre ignorance. comme si nous découvrions que les mots les plus faciles et les choses les plus naturelles peuvent soudain nous devenir inconnus.

Ainsi que l'observe Antoine Berman, la réflexion de Blanchot apparaît ici comme totalement prise dans l'espace littéraire ouvert par l'Athenäum. Les zélateurs de l'Athenäum allaient ainsi poser les jalons d'une théorie (et d'une pratique, ce qui est ici. suivant l'angle d'attaque primé par ces Romantiques, la même chose. s'agissant d’abord de Dichtung. d'une poélique généralisée) de la traduction qui trouvera son ultime aboutissement dans l'étude d'une remarquable profondeur, certes aussi de pénétration difficile, que nous a léguée Walter Benjamin avec "La tâche du traducteur" (Die Aufgahe des Ühersetzers, 1923). Si, en effet, l'acte de traduire a pu exercer sur les Romantiques pareille fascination, celle-ci pourtant, comme le souligne A. Berman.

... ne concerne en aucune façon le rapport des langues entre elles, mais ce qui, dans toute traduction, concerne la mise à mort d'un langage naturel et l'envol de l'ceuvre vers un langage stellaire qui serait son pur langage absolu. La théorie de la traduction de Walter Benjamin. inconcevable sans son long commerce avec les Romantiques. ne fait qu'énoncer plus purement les intuitions de ceux-ci.

On voit donc comment la théorie de la Kunstsprache (tout comme celle de la versabilité infinie de la poésie universelle progressive et de l'Encyclopédie ) invite secrètement à une théoric de la traduction: dans une telle optique, toute aruire est traduction, soit rersion! indéfinie de toutes les formes textuelles et catégorielles les unes dans les autres, soit infiniti. sarion des "mots de la tribu". Ce que l'on considère habituellement comme la négativité de la traduction est dès lors, pour l'Athenäum, bien plutôt sa positivité pó́tique. (A. Berman $1984: 1611$

Si l'œuvre à traduire est achevée dans sa Faktur (expression de Novalis) originale. elle est toujours en gésine, toujours au creuset chez le traducteur. Dès lors, suivant la théorie élaborée progressivement par les Romantiques d'Iéna, celle précisément de la traduction comme potentialisation absolue de l'cuuvre a traduire, cette dernière serait investie d'une «tendance», ou encore animée par une visée a priori, eu égard à laquelle "l'Idée de l'Euvre que l'ceuvre veut être, tend à être (indépendamment ou non des intentions de l'auteur), mais empiriquement n'est jamais» (ibid. : 172). A. Berman relève un premier paradoxe qui n'en est pas un si l'on se rend aux postulats de nos Romantiques. qui sont tout à fait limpides. donc qui ne s'avèrent contradictoires qu'en regard d'une cer- 
taine appréciation de l'acte de traduire, qu'on peut qualifier de "canonique», et selon laquelle il y aurait nécessairement une perte, ou une entropie du signifié dans le transfert entre la langue-source et la langue-cible. C'est tout le contraire qui se donne à entendre ici puisque, contre toute attente, la visée a priori soutenue par l'Úhersetzung romantique "produit nécessairement un 'meilleur' texte que le premier. ne serait-ce que parce que le mouvement de passage d'une langue à l'autre. l'Ühersetzung, a nécessairement éloigné, écarté de force l'œuvre de cette couche empirique première qui la séparait de sa propre Idée : en d'autres termes, l'auvre traduite est plus proche de sa visée interne, et plus éloignée de sa pesanteur finie" (ihid.).

Mais il n'est pas dit pour autant que ce soit là un tenant qui ne trouve sa résonance que dans le cadre de la spéculation romantique. puisqu'il se peut vérifier dans maints spécimens de traduction littéraire. Un exemple éloquent à cet égard, mis en relief par George Steiner dans After Babel, est la traduction d'un poème de Jules Supervielle par Paul Celan, qui ne l'a pas simplement «tiré» vers l'allemand, mais a littéralement procédé à une recréation poétique supérieure à l'original. La redistribution des scansions, mais encore le point d’équilibre ménagé par la gestion de la césure et des procédés d'inversion et d'allitération se résout dans une facture rythmique qui n’a pas son égal dans la livraison originale du poème. On peut donc dire que Celan a dé-naturé (ici au sens de l'opposition entre "Natur" et "Faktur" chez. Novalis), voire dé-créé ou dé-réalisé le poème de Supervielle pour l'élever à sa potentialité propre (Steiner 1975 : 4(14-405). Dans le même ordre d'idée, on a la remarque formulée par Jorge luis Borges lors d'un entretien sur la traduction, où il se rappelle que Chesterton avait dit que bien qu'il ne connaisse pas la langue persane il pouvait néanmoins affirmer que la traduction du Rubáiyát proposée par Fitzgerald constituait un trop beau poème pour être fidèle à l'original'.

C'est dire que l'ceuvre n'atteint la profondeur insoupçonnée de sa plus intime dimension et ne déploie le faisceau de «mondes possibles» qu'elle recèle dans son frayage le plus discret que dans l'acte de traduction. Dans "La tâche du traducteur", prenant en quelque sorte le relais de la Bildung romantique. Walter Benjamin affirme : "Übersetzung [la traduction et non une traduction, comme le traduit de Gandillac) ist eine Form» et. plus en aval, il précise encore qu'entre toutes les formes «celle qui lui revient le plus proprement est de mettre en lumière la post-maturation de la parole étrangère, les douleurs obstétricales de sa propre parole" '1". Qui plus est. une fois saisie sous l'angle de ce processus de potentialisation où s'instruit le «branle» de la traduction, il appert que la poétique même de l'œuvre est déjà nourrie par une pluralité interne. encore indécelée, qui se voit actualisée, comme mise en abyme dans le rapport à l'étranger, rapport qui ouvre cet espace de résolution du sens qui n'est plus saturé ou policé par la fixation et la conservation de la référence ou du signifié pseudo-objectif, mais libère ce point de fuite polysémique qui répond en l'occurrence à un processus d auto-traduction comme passage de l'ceuvre au-delà d'elle-même - se'tzt sich ühe'r, qui est le sens même de la tra-duction comme Über-setzung.

C'est pourquoi aussi celte constellation intime de l'ceuvre, sa «pollinisation" si l'on peut dire en empruntant le terme à Novalis, en appellera pour les Romantiques d'Iéna à la constitution d'une langue «stellaire», préfigurant la notion de reine Sprache chez. Walter Benjamin. Mais, derechef, il ne s'agit pas ici de céder à l'exaltation d'un idiome monologique absolu, donnant dans le subliminal ou l'oraculaire par exemple. L'amplification spéculative promue par la théorie critique élaborée par les collaborateurs de l'Athenäum est pour ainsi dire pré-structurée par cette tension disséminale ou diasporique que $j$ 'ai évoquée plus en amont, bref cette dimension dialogique sui generis qui répond à un état de métamorphose latent implicite à la gestation même de la langue autochtone : il n'y a pas de langue finie. Autrement dit, une langue n'est jamais encore pleinement 
grammaticalisée, et sa lexicalité ou la thésaurisation des nébuleuses tropologiques qu'elle saurait générer ou héberger par acculturation voit le concept d'une sémantique en forme de dictionnaire incessamment débordé, délité et transgressé au profit d'une sémantique conçue en forme d'encyclopédie, tel que l'a fort bien démontré Umberto Eco"l.

Mais c'est dire alors que le contact avec l'auvre étrangère "ouvre" (dans le double sens d' "ouvrir" et d' "ouvrer") le corps de la langue dite "maternelle". lui révèle son propre inachèvement comme levier de sa propre potentialisation. De là, j'en viens à une première thèse que je défendrai formellement dans ces pages : l'impossibilité de la traduction est sa condition de possibilité. En fait, nous rejoignons ici le second paradoxe relevé par Antoine Berman dans le passage du premier au second Romantisme :

... cel étrange destin par lequel ceux qui ont affirmé la traduisibilité a priori de la littérature ont donné naissance à une poéfique de l'intraduisible, poétique bien moins innocente qu'il $n^{\prime}$ y paraît au premier abord, puisqu'il ne peut s'agir, en dernier ressort, que d'une poétique régressive de l'incommunicable. Cette poétique s'imposait nécessairement én rabsence de toute théorie positive du langage naturel. (A. Berman 1984 : 191)

Mais cet «étrange destin" n'est peut-être que la rançon de notre condition postbabélienne, à savoir la précession inentamable de la dotation plurale (et chorale sans doute) des «langues" sur toute appréhension ou intelligence du "fait" de langage comme procès de symbolisation et d'encodage assurant la mémoire, l'intégration et la gouverne de notre expérience en relation avec l'émergence d'une communauté de droit à laquelle des agents linguistiques sont appelés à participer sur la base d'un assentiment minimal à l'exercice de la mutualité, de la réciprocité et de l'équité. Ce state of affairs, le fait proprement inscrutable que la pluralité des idiomes précède et ne laissera jamais de précéder l'analyse qui saurait en être proposée, a été pris en vue par Jacques Derrida. Dans l'une de ses gloses particulièrement décapantes sur la poétique babélienne de Joyce, il remarque d'abord que «l'affirmation d'une langue par elle-même est intraduisible. L'acte qui, dans une langue, remarque la langue même, l'affirme ainsi deux fois, une fois en la parlant, une fois en disant qu'elle est ainsi parlée ; il ouvre l'espace d'une re-marque qui à la fois, du même coup double, défie et appelle la traduction". Cet écart singulier qui joue déjà dans le rapport du locuteur indigène à sa "propre» langue détermine en retour ce qui déjà le détermine sous ce rapport, soit le rapport à l'étranger, de telle sorte que «ce qui reste intraduisible est au fond la seule chose à traduire, la seule chose raductible. L’à-traduire du traductible ne peut être que l'intraduisible»12.

Délier cet écheveau est déjà tout un programme. Cette remarque sur la re-marque de la langue confrontée a l'impropriété même de ce qui lui serait le plus propre est en quelque sorte le «précipité» d'un plus ample développement que Derrida consacrait à l'essai de Benjamin, Die Aufgabe des Übersetzers, dans un texte paru en 1985 sous le titre «Des tours de Babel». C'est donc sous l'égide lézardée et fracassée du nom de Babel, un nom propre en déconstruction qui dénote l'impropriété du nom. que s'engage cette poursuite de l'essence même de la dispersion, de cet emblème, de ce blason fracturé dont les débris vibrent dans le corps plural des langues. Il sera question ici de don et de dette, comme c'est souvent le cas dans les textes de Derrida parus depuis 1985 (bien avant, en fait). Don et dette il y a en effet, et déjà le titre de l'essai de Benjamin fait référence à cette constellation sémantique dans le vocable Aufgabe, qu'on traduit par la «tâche», la «mission» ou le «devoir» qu'il incombe au traducteur d'assumer. J'ai moimême proposé ailleurs de traduire ce titre par l'«abandon" ou la "dédicace" du traducteur ${ }^{13}$.

Don et dette il y a, puisqu'il y va pour Benjamin dans le «traduire», dans l'übersetzen, d'un «survivre», d'un Überleben, de quelque chose de vivant qui aurait une vie 
devant soi, à la limite hors de soi - quelque chose qui se heurte à une frontière, au franchissement. au passage de ce qui a une vie propre vers un seuil qui annonce une autre vie qui a aussi sa vie propre. Mais pour Benjamin cette notion de "survie" doit être soustraite à son concept purement organique pour être restituée au giron de l'histoire où le concept de vie rencontre sa pleine et plus intense extension. C'est pourquoi aussi, comme le note Derrida, le lien ou l'obligation de la dette contractée par le traducteur ne passe pas entre un donateur et un donataire mais entre deux textes. La traduction comme "survie» interdit que son opération puisse être simplement confinée à un exercice de réception, pas plus qu'elle n'a pour destination essentielle de communiquer quelque chose. Cette opération ne saurait enfin se traduire par une duplication ou une reproduction, puisque le rapport de l'original à sa version anticipée doit être maintenu comme rapport, frappant ainsi de caducité l'usufruit de la dyade canonique constituée par les notions de langue-source et de langue-cible. Seule reste l'exigence, voire le commandement, l'injonction signifiée par l'original qui dicte la forme. cette "Form" qu'est déjà la traduction dont l'Aufgahe creuse le passif de sa dette à l'endroit du don des langues (Gabe der Sprache) et de la dotation du nom (Gebung des Namens). Mais pareille forme n'a rien d'un concept générique balisant le divers de la manifestation pour l'ordonner à un appareil catégorial assurant la gestion de l'univocité dans le renvoi de signifiant à signifié. La résistance marquée par l'unicité de l'œuvre, mais encore l' "opacité référentielle» dont d'aventure elle serait porteuse en cela même qu'elle oblige à considérer sans pouvoir s'en abstraire cette mise en abyme où se dessine le hiatus entre ce qu'elle communique et le fait qu'elle communique quelque chose qui n'est pas un contenu mais la pure communicabilité, correspond en quelque sorte, comme le note Derrida, à :

... la loi intérieure de l'original. Celui-ci evige la traduction même si aucun traducteur n’est la. en mesure de répondre à cette injonction qui est en même temps demande el désir dans la structure même de l'original. Celte structure est le rapport de la vie a la survie. Celte exigence de l'autre comme traducteur. Benjamin la compare a tel instant inoubliable de la vie: il est vécu comme inoubliable, il est inoubliable même si en fait l'oubli finit par lemponter $|\ldots|$ De même, l'exigence de la traduction ne souffre en rien de n être pas satisfaite: du moins ne souffre-t-elle pas en tant que structure même de l'auvre. En ce sens la dimension survicante est un a priori - et la mon n'y changerait rien 14

Quelque chose ici se donne et se diffère comme la post-maturation (Nachreife) d'une semence qui en appelle à une obstétrique dont la matrice est le temps, lequel affiche aussi comme exposant ou dans son coefficient un principe de mort que la traduction a charge d'inverser : pour Benjamin il s'agit de transmuer la structure de deuil, le devenir-fossile de l'original en une forme vouée à une croissance dont le passif pèse tout entier sur le centre de gravité du traducteur. Et parlant de gravité, «rien n'est plus grave qu'une traduction", écrit Derrida, "Car si la structure de l'original est marquée par l'exigence d'être traduit, c'est qu'en faisant la loi l'original commence par s'endetter aussi à l'égard du traducteur. L'original est le premier débiteur. le premier demandeur, il commence par manquer - et par pleurer après la traduction” (J. Derrida 1987b: 218). Pleurer après la traduction? Derrida évoque ainsi la condition débitrice de YHWH, après avoir "semé" la confusion, après que Babel a été terrassée, sapée en son fondement, pour devenir l'emblème de la dissémination affectant au tout premier chef son propre Nom imprononçable, car :

... en donnant son nom. Dieu en a aussi appelé à la traduction. non seulement entre les langues devenues tout à coup multiples et confuses, mais d'abord de son nom. du nom qu'il a clamé, donné, el qui doit se traduire par confusion pour être entendu, donc pour laisser entendre qu'il est difficile de le traduire et ainsi de l'entendre. Au moment où il impose et 
oppose sa loi a celle de la tribu, il est aussi demandeur de traduction. Il est aussi endetté. Il n'a pas fini de pleurer après la traduction de son nom alors même qu'il l'interdit. [...] Comme dans La folie du jour, la loi ne commande pas sans demander d'être lue, déchiffrée, traduite. Elle demande le transfert (Übertragung et Übersetzung et Überleben). Le double bind est en elle. En Dieu même, et il faut en suivre rigoureusement la conséquence : en son nom. (/hid. : 218-219)

Cette condition débitrice répond ainsi à la contraction d'un double bind, d'une «double ligature» qui noue l'un a l'autre des noms propres, le nom propre étant l'intraduisible même qui survit au bord de «la» langue. Car son référent est unique, mais cette unicité est telle qu'elle offusque le jeu de la référence comme instance appelée à valider l'occurrence vérifiant une propriété partagée par une classe d'individus : la «propriété" unique de ce nom marque l'impropriété de ce qui en fait un nom "propre», n'étant pas un prédicat de l'individu qu'il dé-nomme, ruinant le renvoi de signifiant à signifié, l'écart présumé par l'articulation du concept à son référent. L'impropriété du nom propre bordant l'in-oui de la langue désigne le lieu même de la traduction, ce en quoi celle-ci ne peut qu'aggraver sa condition débitrice, celle de la langue "propre» creusant l'inachèvement de sa propre facture dans le rapport à l'étranger qui s'endette en enjoignant son débiteur : "La dette $n$ 'engage pas de sujets vivants mais des noms au bord de la langue ou. plus rigoureusement, le trait contractant le rapport dudit sujet vivant à son nom, en tant que celui-ci se tient au bord de la langue. Et ce trait serait celui de l'à-fraduire d'une langue à l'autre. de ce bord à l'autre du nom propre" (ibid. : 219). Il y va donc ici, ainsi que Derrida nous le donne à entendre, d'un contrat tout à fait singulier, scellé sous le signe de l'unicité sans unité préalable qui lui conférerait une assise en dehors de sa contraction. de son exposition à l'autre, où la langue «maternelle» contracte sa propre limite. cette frontière intérieure à sa propre gestation.

Il n'y a pas de métalangage qui vienne assurer la mise en dehors de sa condition débitrice. Et il en est ainsi parce que avant le langage, il y a les langues. la (jahe der Sprache qui en appelle à l'Aufgabe du traducteur. C'est pourquoi dans la traduction il y va toujours de la traduction, de sa possibilité comme fiduciaire de son impossibilité. En effet, la traduction «ne chercherait pas à dire ceci ou cela, à transporter tel ou tel contenu, à communiquer telle charge de sens mais à re-marquer l'affinité entre les langues, à exhiber sa propre possibilité» (ihid.). Benjamin établit ainsi le passif de cette inadéquation qui détermine la possibilité même du "traduire" comme survie. c'est-à-dire en tant que rapport qui ne se résout pas dans quelque principe de clôture saturant l'ébauche d'une équivalence virtuelle entre les idiomes, mais qui ne laisse de se radicaliser en tant que rapport nourri par la convergence secrète de ces idiomes, laquelle est tout entière instruite par la tension de ce rapport, par son intensité :

Il est impossible qu'elle fla traduction| puisse révéler ce rapport caché lui-même. qu'elle puisse le restituer |herstellen] : mais elle peut le représenter [darstellen] en l'actualisant dans son germe ou dans son intensité. Et cette représentation d'un signifié [Darstellung cines Bedeuteten] par l'essai, par le germe de sa restitution, est un mode de représentation tout à fait original, qui n'a guère d'équivalent dans le domaine de la vie non langagière. Car cette demière connaît, dans des analogies et des signes, d'autres types de référence |Hindeurung| que l'actualisation intensive, c'est-à-dire anticipatrice, annonciatrice lvorgreifende, andeutende|. Mais le rapport auquel nous pensons, ce rapport très intime entre les langues, est celui d'une convergence originale. Elle consiste en ceci que les langues ne sont pas étrangères l'une à l'autre, mais, a priori et abstraction faite de toutes relations hıstoriques, sont apparentées l'une a l'autre en ce qu'elles veulent dire. (W. Benjamin $1971: 264$ ) 
Ce rapport caché, cette affinité secrète, $n$ 'est pas étrangère à la Potenzierung évoquée par les Romantiques d'léna : l'original est en gésine, au creuset dans l'inachèvement de sa forme qui en appelle à une "rédemption" par l'étranger qui ne peut l'encrypter sans le décrypter, l'inséminer sans se disséminer. L'actualisation de ce rapport asymétrique oblige tant et si bien que le traducteur doit lui-même s'en absoudre en marquant l'impossibilité de cette absolution qui voit toujours s'accroître sa dette comme passif inscrit dans sa langue même et qui détermine en l'occurrence la croissance d'une semence appelée à s'absoudre de sa condition générique pour contracter le chiffre de son incidence temporale, l'unicité de sa marque, sa cicatrice. Pareille signature interdit que l'original puisse simplement trouver un équivalent dans sa traduction, en sorte que le heurt frontalier qui sévit entre les langues répond plutôt à un frayage néguentropique qu à une espèce de fatalité empirique grevant la «blanche écriture» du concept qui aurait charge d'en lessiver les scories. "Racheter dans sa propre langue ce pur langage exilé dans la langue étrangère, libérer en le transposant ce pur langage captif dans l'ouvre, telle est la tâche du traducteur. La traduction est transposition poétique (Umdichtung)" (W. Benjamin 1971).

L'impossibilité qui est assumée par cette Umdichtung sans que jamais elle sache s'acquitter du passif de sa dette qui devient par son déficit même l'élément séminal de la "survie» de l'original, commande la métaphorique nuptiale et obstétricale mobilisée par Benjamin et qui se résout dans la composante nucléaire et épidermique du fruit qui s'offre mais qui ne sera jamais complètement consommé, puisque la cohésion de la "teneur» (Gehalt) de l'original et de la Sprache à laquelle elle se trouve intriquée, expose le traducteur à l'inexhaustivité du matériau qui "mande» son exercice sous le coup d'une résistance qui sollicite et interdit à la fois et qui. pour Benjamin, caractérise l'approche infinie du royaume de la reine Sprache, du "pur langage". La constellation métaphorique du fruit et de l'enveloppe. du noyau et de l'écorce réfêre pour l'essentiel à l'imprégnation discrète de motifs kabbalistiques qui courent en filigrane dans l'essai de Benjamin, marquant ainsi sa déférence à une eschatologie messianique qui cependant, che\% lui, s'avère pleinement sécularisée.

Plus précisément on peut définir ce noyau essentiel comme ce qui. dans la traduction. n'est pas a nouveau traduisible. Car, autant qu'on en puisse extraire du communicable pour le traduire. il reste toujours cet intouchable vers quoi s'oriente le travail du vrai traducteur. Il n'est pas transmissible comme l'est la parole créatrice de l'original lähertraghar n'ie' das Dichterw'ort des Originals]. car le rapport de la leneur au langage est tout à fait différent dans l'original et dans la traduction. Dans l'original, teneur et langage forment une unité déterminée, comme celle du fruit et de l'enveloppe : le langage de la traduction enveloppe sa teneur comme un manteau royal aux larges plis. (W. Benjamin $1971: 268$ )

Cet «intouchable» (unheriührbar) n’a rien d'ésotérique : il est là. devant, dans la «facture» de l'original qui en appelle à une rédemption qui pourtant jamais ne l'absoudra suffisamment pour que le roi ou la reine trouve un manteau qui lui sied définitivement. L'incomplétude de toute traduction est le ressort même de sa nécessité, son indigence le facteur même de sa croissance, son impossibilité, sa condition de possibilité.

Le royaume est tout près, qui hée mais demeure inviolable, comme dans la parabole de Kafka. Devant la loi, où le néophyte demeure interdit devant une porte désespérément ouverte.

L'approche infinie du royaume signifie qu'il est à portée de l'aspirant(e) mais lui enjoint d'aspirer toujours davantage dans le sens de la déchirure, de la lésion qui ouvre le corps plural, utérin oserais-je dire, des langues qui font que notre condition postbabélienne est une bénédiction dont il appartient au traducteur de mesurer l'ampleur et la 
portée dans la livraison métisse des ressources idiomatiques où le mortel tente de retracer l'Orient dés-astré - suivant ici l'expression de Maurice Blanchot - de son errance première.

Tel un funambule franchissant le pas entre les plaques tectoniques où sont burinées les cicatrices de sa mémoire récente hantée par les lueurs fossiles d'astres éteints depuis des milliards d'années-lumière, celui (celle) qu'Aristote désigne comme le "vivant doué de parole lzoion logon ekhon|» ne laissera ainsi de réouvrir le pacte herméneutique qui le lie au partage d'un ckoumène désormais ouven à l'étrangeté absolue du visage humain. dont la déchirure unique dessine en tout un chacun une origine autre du monde qui n'a d'autre refuge que l'exil dans la parole tenue, promise, donnée, et perdue sans aucun doute. Derechef, rien d'ésotérique ici : c'est là, faut-il le dire, et dans l'exclusive de toute tangente confessionnelle, le sens même de la judéité de l'homme, du frayage de cette "faible force messianique" évoquée par Walter Benjamin dans ses Thèses sur la philosophie de l'histoire, rédigées peu avant qu'il soit contraint au suicide, à la frontière espagnole, en 194015.

Être à portée du royaume sans pouvoir y entrer. sans espoir même d'en contempler le seuil. c'est maintenir ouvert le rapport entre la diaspora des langues et le frayage «fugitif» (flüchrig) de cette reine Sprache certes à jamais disséminée dans la multiplicité des formes où le mortel accède à l'expression, mais qu'on ne saurait forclore au profit d'une économie transcendantale dont le magistère est d'ores et déjà en déficit de sá propre déshérence. Le mortel. bref le sujet parlant qui s'exprime loujours de quelque façon depuis l'imminence de sa propre mort, ne peut jamais s'abstraire de l'expression où il tente de cristalliser l'apprentissage de sa propre condition, qui demeure toujours celle d'un nourrisson à l'abandon. du sujet infans évoluant à tâtons à travers les nébuleuses du microcosme linguistique qui le soude à l'aura maternelle, quand son babil hanté par l'icône spéculaire où il risque de s'emmurer vivant s'investit du sort de Babel, c'est-àdire apprivoise l'éloignement croissant de ce paradis à jamais perdu où le pleur très ancien de l'animal s'est prématurément involué dans le néant de la forme grammaticale qui satellise l'émoi premier du petit d'homme dans un champ gravitationnel qui $n$ 'admet d'existence qu'elliptique et subliminale, ne laissant ainsi d'errer sur la "nef des fols" qui nous a vu(e)s échoir dans les archipels de la parole. On songe entre autres ici à cette clef heuristique indispensable que Jacques Lacan nous a tendue dans Le stade du miroir 16.

Il est certes paradoxal qu'un être prématuré comme le mammifêre humain, qui est déjà en déficit dans l'usage même de sa langue «maternelle», puisse aussi «mettre en lumière la post-maturation de la parole étrangère, les douleurs obstétricales de sa propre parole», comme l'écrivait plus haut Benjamin. Le mortel confronté à celte amplitude abyssale, qui est la condition même de son rapport à la langue, ne peut que s'approcher de la matrice originelle de son expression sans jamais toucher au terme qui réfléchirait la source de ce qui s'exprime en elle : l'Aufgabe du traducteur est à l'image de cette condition apatride, une passion de métèque qui s'endette toujours davantage par-devers ce don prodigue qui pratique une brèche dans sa propre langue, et dont il ne peut courtiser que la forme.

Aussi, que l'abîme entre nos langues orphelines de toute origine soit mis en abyme dans le blason fracturé de cette langue «stellaire" appelée de ses voux par la spéculation romantique. est à la mesure même de ce frayage diasporique qui confronte l'acte de traduction à sa propre impossibilité et qui l'instaure comme telle dans sa nécessité même, en l'occurrence comme rapport ne pouvant être mené à forclusion en guise de translation entre une langue-source et une langue-cible. Un rapport demeuré rapport. C'est précisément la leçon que dégage Henri Meschonnic dans la très belle étude qu il a consacrée à la question de l'allégorie chez. Benjamin, y retraçant le sens même. sinon l'une des avenues 
empruntées dans l'expression de sa judéité. Qu'il s'agisse des agrégats de citations négociant divers passages entre les débris babélisés de la modernité baudelairienne, ou de l'analyse micrologique (dans le sens primé par Adorno) de la fragmentation de la mémoire fulgurée dans la Jetzt-Leit, le hiatus instantané d'une dialectique en suspens. la méthode inventée par Benjamin, écrit Meschonnic, «produit, par le rapprochement des brisures, une rythmique de signifiance, qui est présente el n'est pas représentée. De même, la tâche du traducteur est de produire un passage qui reste passage. Non, comme le veut l'idée courante, une arrivée, mais un rapport demeuré rapports'l?.

Comment situer ce rapport dans l'amplitude abyssale entre la disparité foncière des idiomes et l'appel à l'épiphanie d'une reine Sprache constituant la visée a priori du traducteur dans son travail d'obstétrique ? Comme Derrida le stipule. Benjamin distingue soigneusement le mode de la visée (die Art des Meine'ns) et la chose visée (das Gemeinte). C"est le mode de la visée qui importe ici. puisque la chose visée le sera toujours selon des modes différents en toute langue donnée et en chacun des spécimens qu'elle porte à l'attention du traducteur qui ne peut que faire l'épreuve de la distance à partir de l'inachèvement même de sa propre langue. Ce qui est visé par la traduction, c'est "la langue même comme événement babélien, une langue qui n'est pas la langue universelle au sens leibnizien, une langue qui n'est davantage la langue naturelle que chacune reste de son côté, c'est l'être-langue de la langue, la langue ou le langage en tant que tels, cette unité sans aucune identité à soi qui fait qu'il y a des langues, et que ce sont des langues» (J. Derrida 1987b : 232-233).

L'implication d'une "tonalité» messianique dans le propos de Benjamin n'est pas fortuite. Non seulement s'agit-il de veiller et de vaquer à la croissance ou à la «renaissance $[$ Auflehen] infinie des langues" à travers l'«éternelle survie des ceuvres [am ew'igen Fortlehen der Werk'(')», mais le texte sacré détermine le lieu même de la traduction, son extraterritorialité aussi bien que sa nécessité qui, je l'ai déjà signifié, est fonction de son impossibilité. Le "savoir de cette distance", nous dit Benjamin. l'Enffernung. l'éloignement absolu qui détermine le rapport au texle sacré, marque la limite de la traduction et son assignation en guise de rapport demeuré rapport, bref comme "le mode 'intensif" qui rend présent ce qui est absent, laisse venir l'éloignement comme éloignement, fort : da» (ihid. : 234). Si la traduction rencontre sa limite et sa possibilité même là où le canonique en appelle à la transgression. comme YHWH lui-même en appelle à la traduction de son tétragramme imprononçable, c'est que la traduction s'y voit extradée à l'interface entre l'injonction implicite qui structure la teneur révélée des vocables destinés à une écoute "créaturiale" et la libéralité de l'intelligence rompue à sa finitude el qui ne laisse ainsi d'apprivoiser l'abandon extrême de sa condition dans la distance au texte qui fournirait le sens plénier, mais jamais réalisé, de cette condition, dans sa dissémination en guise de "faible force messianique" frayant sa voie au sein de la virtualité du sens pollinisé dans la tension intralinéaire des versions où s'organise sa "survie", qui est mise en abyme comme condition sine qua non du rapport au texte sacré.

Jamais il n'y a plus traductible, mais en raison de cette indistinction du sens et de la littéralité (Wörtlichkeit), le Iraductible pur peut sannoncer, se denner, se présenter, se laisser traduire comme intraduisible. Depuis celte limite, à la fois intéricure et extérieure. le traducteur en vient à recevoir tous les signes de l'éloignement (Enternung) qui le guident en sa démarche infinie, atu bord de l'abime, de la folie el du silence : les dernieres ceuvres de Hölderlin comme traductions de Sophocle, l'effondrement du sens "d'abîme en abîme" : ce danger n'est pas celui de l'accident, ce'est la traduction. cest la loi de la traduction, là-traduire comme loi. l'ordre donné. l'ordre reçu - et la folie attend des deux côtés. Comme la tâche est impossible aux abords du texte sacré qui vous l'assigne, la culpabilité infinie vous absout aussitôt. (Ihid.) 
Mais ce qui sévit dans l'abord du texte sacré, cette folie qui attend des deux côtés, sous ces angles multiples qui se découvrent et foisonnent quand nous tentons d'approcher la source qui de si loin. depuis si longtemps, nous aura déportés, nous transit avec une rigueur non moins pénétrante dans le commerce avec l'œuvre littéraire. Son caractère profane, plutôt métis en tant que le sacré et le profane y convolent à une frontière dont les bornes sont davantage des gouffres que des étalons. s'avère parfois plus violent et unheimlich que l'aura qui garde les abords du texte sacré. Cela tient au caractère de l'œuvre en tant qu'œuvre. Le caractère de l'œuvre en tant qu'cuvre traduit l'irréparable. À cet égard, on peut s'en remettre ici à l'axiomatique lapidaire et incisive qu'a dégagée Giorgio Agamben dans son remarquable $L a$ comunita che viene. où l'on peut lire: «Révélation ne signifie pas révélation du caractère sacré du monde, mais seulement révélation de son caractère irréparablement profane.» Et plus loin encore : «La rédemption n'est pas un événement où ce qui était profane devient sacré et ce qui avait été perdu est retrouvé. La rédemption, au contraire, est la perte irréparable du perdu, la profanation définitive du profane»18.

L'irreparable, la profanation définitive du profane, signifie que nous habitons désormais, depuis toujours peut-être, les coutures d'un texte orphelin de toute origine. sous ses ratures et dans les codicilles qui y sont appendus comme des épaves arrachées à l'océan d'une mémoire hantée par l'étemel ressassement du protocole commandant l'accès au sens disséminé dans ces lambeaux maculés de sémaphores et saturés d'un silence plus impénétrable que le mutisme dans lequel la mon enveloppe le lourd dividende, le tribut implacable qu'elle prélève à chaque génération.

Les individus qui peuplent les univers de Kafka, de Genet, de Beckett, de Pirandello, de Carlo Emilio Gadda, de Faulkner, el celui encore de Rejean Ducharme. sont des saints. Sauf qu'il $n$ 'y a pas de ciel et qu' après Auschwitz, l'enfer est une colonie de vacances. La langue qui est travaillée par cette déshérence, la concentration. tantôt paroxystique tantôt diffuse, de l'irréparable qui irradie l'opacité secrète de l'œuvre. assigne le lieu même de la traduction, bref, lui enjoint la gouverne frontalière d'un matériau délibérément polysémique, parce que sa rédemption ne vient pas de l'unité de la forme, mais de son unicité, d'un décret unique qui force la langue qui en appelle à sa propre expropriation. L'unicité de la facture à laquelle tient l'intime étrangeté de l'ceuvre, ce qu'elle porte en elle d'incommunicable par rapport à la langue où elle s'exprime. celle langue qu'elle déserte et qu'elle fouit à la fois, la situe déjà dans une extraterritorialité que les ressorts de la traduction vont développer de façon exponentielle. Du point de vue de l'analyse linguistique. plus précisément de l'analyse logique et grammaticale, que la traduction soit possible est un fait. Mais aussitôt qu'elle est déportée sur le plan de l'ouvre littéraire, là où la «littéralité» vient directement en question, la traduction devient inévitable dans son impossibilité même, essentielle parce que impossible. Pourquoi? C'est que le rapport profond, pour ne pas dire abyssal, qui lie l'ceuvre en tant qu'ceuvre à la traduction consiste, comme le précise Antoine Berman, «en ce que l'œuvre, en surgissant comme ouvre, s'institue toujours dans un certain écart à sa langue». Si, en effet, la traduction accomplit l'ceuvre au-delà de sa facture originale, c'est-à-dire la pousse audelà d'elle-même, en revanche :

... cette "aliénation" est déjà préfigurée dans son rapport à sa langue d'origine. L'étrangeté native de l'ceuvre se redouble de son étrangeté (effectivement accrue) dans la langue étrangère. Et c'est que la traduction est pour elle une véritable mélamorphose, une réelle Veränderung - et cela d'autant plus que cette dernière est plus fidèle. plus "elittérale». [...] Ce mouvement par lequel l'ceuvre devient "mythique». c'est elle-même qui le permet : ou, en d'autres termes, l'aure est cette production par laquelle la traduction deviemt wise activité pleine de sens. (A. Berman 1984:201-202) 
En quoi, pour suivre le fil de ce paradoxe, la littéralité serait-elle inductrice d'étrangeté ou, si l'on préfère, en mal de métamorphose ? Prenons la littéralité absolue : Pierre Ménard copie méticuleusement deux chapitres du Don Quijote de Miguel de Cervantes et publie cette "version" en soutenant qu'elle est supérieure à l'original. Borges, à qui l'on doit ce chapitre remarquable dans l'intelligence de l'«entité» littéraire, soutient à travers son narrateur que cette version tauto-logique est en fait plus complexe que l'original : le calque sélectif, l'attrition impliquée par sa stratégie, devient un facteur de complexification qui se double d'un anachronisme patent pour un écrivain français du début du $X X X^{c}$ siècle. Pierre Ménard n aurait pu produire par ses seuls moyens une telle facture, même en procédant à de multiples ébauches. Nous avons ici une traduction dont le vecteur, aussi bien que le matériau, est le temps. L'écart, l'effet d'anamorphose ou encore, pour puiser ici au lexique de l'astronomie, cet effet de "parallaxe" (déplacement de la position apparente d'un corps, dû à un changement de position de l'observateur) nous introduit de quelque façon aux abords d'une reine Sprache dont la version de Pierre Ménard exhibe un angle singulier en re'-marquant l'unicité de la poétique de Cervantes. qui est ici cristallisée par l'effet d'une spoliation chirurgicale. Cette transmutation discrète nous permet de discerner sur le plan de la plus stricte littéralité ce en quoi la résistance de l'oxuvre, l'unicité de sa facture, en appelle à la traduction et l'oblige à émerger comme opération sui generis, quelle que soit sa dépendance à l'égard des instances qui la sollicitent : transmission, acculturation, etc. Si certaines techniques ou méthodes de rewriting peuvent être indiquées, sinon «canonisées» du point de vue de l'économie générale de la traduction, en revanche il n'y a pas de formule. Cela se vérifie de façon expresse dans le contact avec cette "masse critique» que constitue l'ceuvre littéraire, qui est pure exposition, en deçà et au-delà de toute proposition véhiculée par sa facture et qui la rendrait ancillaire à la translation d'un contenu dont la référence répondrait aux attentes d'une truth-conditional semantics, en l'occurrence à la réduction des éléments tensoriels de son expression, par exemple la dimension essentiellement "performative" de sa livraison. sa teneur dialogique ou sui-référentielle, au profit d'une structure monologique qui serait garante de l'univocité ou de l'isomorphisme conceptuel assurant le transfert du signifié d'une langue a l'autre. Si la traduction se révèle précisément comme opération sui generis dans l'approche de l'cuuvre littéraire, c'est que celle-ci est essentiellement performative, elle produit de l"uêtre" et oblige : son économie matricielle, le fiat par lequel elle s'instaure comme matrice de «mondes possibles». répond sensiblement à l'énoncé performatif et sui-référentiel "la séance est ouverte». Celle «séance» de l'ouvre ne sied à rien d'autre qu'elle-même. L'ouvure ne (se) prononce pas (sur) la vérité, elle s'exécute, se liquide, se diffère à une lecture qu'elle n'attend pas. car elle sait que Godot ne viendra pas. Mais ça, elle peut le dire, et c'est pourquoi elle est sui-référentielle: elle ne communique rien sinon la communicabilité elle-même. Quelqu'un qui ne communique rien, sinon le fait qu'il communique. se communique. Cette intransitivité de l'exuvre fonde la traduction comme opération sui generis, dans la mesure où a'incommensurable résistance qu'elle oppose à sa traduction - traduction qu 'en même temps elle permet et appelle - donne tout son sens, non moins incommensurable. à celle-cim. (A. Berman 1984:202-203)

Cette contradiction in adjecto ne se résout aucunement dans quelque profession en faveur de l'ineffable. L'étanchéité de la facture est ni plus ni moins qu une façon de prêter le flanc à la rigueur de la traduction dont la démunition, loin simplement d'être rançonnée par la finitude de ses ressources idiomatiques, pourra donner lieu à un mûrissement tout à fait singulier, parfois inattendu, de la forme se dégageant du négoce frontalier impliqué dans le rapport à l'original. Ce rapport est en principe infini. Mais il ne s"agit pas ici de céder à un relativisme tous azimuts. II convient plutôt de s'en remettre à la 
notion d'opera aperta introduite par Umberto Eco : la force de l'œuvre, son «apérité» si je puis dire, s'imposera dans la virtualité des ressources qu'elle éveille dans la pluralité des idiomes mobilisés pour en ménager l'approche et lui prodiguer un aval dans les territoires qui doivent eux-mêmes fonder l'expérience de la traduction, en tant que celle-ci est en quelque sorte justiciable de la dissidence qui est déjà marquée par l'œuvre en regard des limites imparties par le champ de son expression native. C'est pourquoi, en l'occurrence. Shakespeare appelle les "versions" de Michel Garneau et de Robert Lepage, et les Variations Goldberg la "traduction» de Glenn Gould. George Steiner a décrit dans un ouvrage désormais indispensable le vaste laboratoire trans-culturel qui a proliféré autour de l'Antigone de Sophocle'19.

On ne peut décidément plus faire avec un scénario de type translation d'une languesource à une langue-cible. L'espace de la traduction est essentiellement dissymétrique et implique comme tel l'invention de géométries variables dont le vecteur $n$ 'a pas d'abord à se rapporter à une unité de contenu mais à l'unicité de la forme qui migre entre les "versants" des langues qui se traduisent à partir de leur extradition. L'unité du contenu est tributaire de l'unicité de la forme, et non l'inverse. Cette considération, passablement abstraite, peut être décantée et trouver sa résonance dans des contextes pragmatiques nettement différenciés auxquels sont corrélés divers réseaux d'attentes participant de l'horizon herméneutique dans lequel s'inscrit une communauté de locuteurs évoluant à une époque donnée dans une portion de l'okoumène qui leur est dévolue de façon élective ou contingente et qui serait alors l'équivalent d'une niche écologique structurée par une économie symbolique. Dans The Poetics of Translation. Willis Barnstone évoque deux cas remarquables d'extradition qui mettent directement en cause la forme de la traduction par contraste avec la conservation de l'univocité de la référence. Lorsque Bronsilaw Zielinski s'est attaqué à la traduction en polonais du Moby Dick de Melville, if a dû s'acquitter d'une quantité de problèmes pour accoucher d'une traduction qui puisse rencontrer les attentes du texte original et les latitudes qui sont celles de sa communauté linguistique. Si on se déplace plus au nord, on constate aisément qu'il $n^{\circ} y$ a pas d'agneaux qui broutent sur les banquises de l'Arctique. Les traducteurs de la Bible en esquimau ont dû faire l'économie de l'exotisme de l'expression "Agneau de Dieu» et se résoudre à la version aurochtone "Phoque de Dieu»20. De part et d'autre. il ne s'agit pas de vérifier la référence, mais de marquer la prégnance d'un signifié qui n'a d'existence que par le signifiant qui commande la migration du sens à travers divers «mondes possibles" générés par l'impossibilité même de la traduction.

Si. comme je l'ai affirmé plus en amont, l'impossibilité de la traduction est sa condition de possibilité, il appert en retour que celle-ci se détermine en guise de double bind. de "double ligature", suivant la notion introduite par l'anthropologue américain Gregory Bateson dans ses travaux sur la schizophrénie et la cybernétique du soi21. Qu'est-ce qu'un double bind? Une situation de communication où un individu est exposé à deux injonctions contradictoires telles que s'il obéit a l'une, il est forcé de désobéir à l'autre. Il n'est pas difficile d'appliquer cette intrigue à la situation encourue par le traducteur auquel la tradition impute une vocation bifide: traduttore traditore, dans la mesure où il lui incombe de se frayer un passage dans le col de l'abîme entrouvert par l'amplitude entre les ressorts de l'original et les ressources idiomatiques de sa langue maternelle. Mais s'agit-il vraiment de servir deux maîtres? Du reste, y a-t-il quelque chose comme une maîtrise d'œuvre. alors qu'il n'est de métalangage qui vienne coiffer ou subsumer la dissymetrie originelle des langues en traduction? Si le rapport peut et doit demeurer rapport, comme le soutient Henri Meschonnic, il $n^{\prime} y$ aura pas un texte et ses versions, mais des textes qui sont déjà en instance de traduction dans leur facture indigène. 
Dans Women, Fire, and Dangerous Things ${ }^{22}$, le linguiste George Lakoff aborde la question de la possibilité de la traduction du point de vue de la commensurabilité ou de l'incommensurabilité des systèmes conceptuels qui prévalent respectivement dans l'une ou l'autre des langues qui sont candidates à la traduction. Lakoff propose de considérer. outre la description des systèmes conceptuels, ce qu il désigne comme les acapacités conceptualisantes [conceptualizing capacities]» de tel ou tel autre agent linguistique pouvant avoir commerce avec une langue étrangère à celle qui fonde sa compétence indigène. Il en arrive ainsi à une thèse minimaliste dont on ne saurait, à mon sens, faire l'économie : il ne s'ensuit pas de l'impossibilité de la traduction que la compréhension est impossible (G. Lakoff 1987: 311). Lakoff en remet : le partage d'un capital d'expérience de base (basic experience) permettrait à un locuteur d'une langue donnée de comprendre l'autre langue même s'il ne peut la traduire dans la sienne propre. Cela entraîne donc, contre toute attente, que "la traduction puisse être effectuée sans compréhension el qu'il puisse y avoir compréhension sans possibilité de traduire» (ibid. : 312).

Quoique Lakoff $n$ 'en fasse pas mention, cette observation permet de jauger la différence entre la traduction "performée» par un ordinateur, que je désignerai comme transaction ordinale, qui procède d'un réseau d'équivalences entre les token-units appuyées par une générativité syntaxique (arborescente ou rhizomatique) dotée d'une compétence sui-référentielle, et, par ailleurs, la traduction cardinale, dont la gestation engage le facteur humain comme "centre de gravité narratif"2.3 qui, loin de s'en remettre à quelque principe d'adéquation, creuse plutôt l'abîme de la forme sur le mode intensif où s'instruit le rappon sémantiquement non saturé entre la facture de l'original et l'expérimentation des limites de la langue qui s’en enquiert pour s'y traduire en la traduisant au sens fort de la ductio, d’une «insémination» telle que suggérée par Walter Benjamin. S`il peut sembler paradoxal que la traduction puisse être effectuée sans compréhension et qu il puisse y avoir compréhension sans possibilité de traduire, il faut voir aussi qu'il n'y va pas ici simplement d'un cas d'espèce. Par exemple. l'apprentissage d'un texte en hébreu, bref le principe de la lecture de l'hébreu, qui se présente comme un texte originellement non vocalisé, en appelle, comme le souligne Éliane Amado Lévy-Valensi dans La nature de la pensée inconsciente, à des qualités d'intuition qu'on peut rapprocher du principe de l' «attention flottante" préconisée par Freud:

À chaque instant, il faut reconstituer une unité organique du mot situé dans la phrase. alors que l'on ne dispose que de données fragmentaires. La lecture de l'hébreu est comparable a la lecture du syndrome alors que ne sont donnés que quelques symptômes qu il faut relier entre cux. Il y a dans cette lecture le principe même de l'attention flottante. Alors que l'on peut apprendre à lire le grec en une heure et le lire sans le comprendre, l'hébreu non vocalisé implique que l'on comprenne avant de lire. La lecture' cest au terme' du processus e't non à l'origine'24.

Pareille disposition, qu'on peut aussi rattacher à la notion freudienne de Nachträglichkeit, celle d'un "retardement», d'un "après coup" répondant au frayage (Bahnung) en différé des signifiants infra-conscients2.9. donne la mesure de l'important postulat de Benjamin selon lequel «l'élément originaire du traducteur" est le mot et non la proposition ou l'articulation syntaxique, car, écrit-il, «la proposition [Satz] est le mur devant la langue de l'original, la littéralité [Wörtlichkeit] est l'arcade" (W. Benjamin 1971 : 272). L’abîme entre le poétique et le logique est la mesure du langage qui émerge comme expression sui generis nourrie par la dissémination chorale des multiples voix qui instruisent le lieu du sens. Il ne s'agit pas ici de traduire quelque chose dans le langage. mais de se traduire dans une langue qui est toujours déjà en instance de traduction, et i] est clair en l'occurrence que cet exercice transcende l'économie conceptuelle d'une 
sémantique véri-conditionnelle (truth-conditional semantics), où la commensurabilité entre une langue-source et une langue-cible s'appuie sur la préservation des conditions de vérité, dans une traduction «phrase par phrase». La pétition de principe qui forme l'assiette de cette procédure, que $G$. Lakoff rattache au translatability criterion (ibid. : 327) est d'autant plus contestable, précise Lakoff, que la "commensurabilité» n'est pas «un concept naturel ; il est apparu dans le contexte d'expert theories sur le langage et la pensée» (ibid. : 323). C'est là en effet oublier que les langues formelles (ou formulaires) ne sont que «des fragments arbitraires de la langue naturelle» 26 .

L'incommensurabilité entre les «mondes possibles» générés par l'usage de langues nettement différenciées, tant sur le plan de leur morphogenèse qu'eu égard à l'axiologie qui s'y sera greffée au gré de leur évolution respective, ne nous résume pas à quelque forme d' "autisme» linguistique qui ferait de l'inadéquation entre les spectres référentiels corrélés à l'exercice de ces divers idiomes l'avatar d'univers clos livrés à l'arbitraire d'une gravitation pragmatico-sémantique qui interdit toute médiation interculturelle. Aussi, avant même de parler d' «indétermination de la traduction" et d" "inscrutabilité de la référence» au sens où $W$. V. $O$. Quine en a convenu d'un point de vue behaviorial, impliquant alors un réseau d'hypothèses analytiques confortées par des accords pragmatiques $^{27}$, parlerais-je volontiers d'une indétermination de l'intention signifiante, non seulement au niveau de la pluralité des idiomes candidats à la traduction mais dans la production même du signe mobilisé par l'agent présumé maître de son expression dans «sa» langue maternelle. Cette indétermination est la mesure d'une détermination, d'un rapport qui ne se clôt pas sur une corrélation univoque entre intention et signification. mais qui se maintient en tant que rapport, au sens précisément où l'a marqué Henri Meschonnic dans son interprétation de la poétique de la traduction élaborée par Walter Benjamin.

Qu'est-ce à dire ? Le rappont n'est assumé que parce qu'il n'est jamais consommé, et il ne peut l'être parce que ce rapport n'est pas assumable : c'est pourquoi il y a rapport. Rapport demeuré rapport et non point transport ou itération entre une langue-source et une langue-cible. Pareil tenant qui, j'en conviens, n'est pas d'emblée évident, doit être interprété à la lumière de la thèse fondamentale formulée par Benjamin dans son essai Sur la langue en général et sur la langue des hommes (1916). Benjamin en appelle à une conception de la langue qui «ne connaît aucun moyen, aucun objet ni aucun destinataire de la communication», s'agissant alors d'accéder à ce «par quoi plus rien ne se communique, et en quoi la langue se communique elle-même absolument. [...] Il n'y a pas de contenu de la langue : en tant que communication, la langue communique un être spirituel, c'est-à-dire la communicabilité pure et simple» 28 .

À nouveau, rien d'ésotérique ici, puisqu'il s'agit de retracer l'être-langue de la langue, force étant d'abord d'en découdre avec la subordination de l'expression linguistique à quelque chose d'extérieur à elle qu'il lui appartiendrait essentiellement de communiquer en référence au primat de la fonction cognitive enchâssant toute expression douée de sens et qui se conformerait comme telle à la structure sous-jacente de tout énoncé réputé valide. à savoir la forme logique du jugement que l'on porte sur un objet ou un «état de choses» référé idéalement à un ordre objectif de la manifestation, sinon aux offices d'un tuteur transcendantal.

Que le langage puisse exprimer quelque chose, et qu'il le fasse en se conformant aux protocoles et aux attentes normatives régissant l'exercice de l' "agir communicationnel» au sens marqué par Jürgen Habermas et Karl-Otto Apel29, ne fait pas l'ombre d'un doute. Mais l'expressivité même de la langue, l'être-langue de la langue demeure insaisissable en dehors du frayage diasporique des idiomes qui sont toujours déjà en instance de traduction et qui ne révèlent l'unicité de leur forme. par contraste avec l'unité 
du contenu qu'elles sauraient consigner ou véhiculer, qu'en évoluant sous le coup de cette extradition originaire qui répond à la condition post-babélienne de tout locuteur d'une langue donnée pouvant être appelé à «mettre en lumière. comme l'écrit Benjamin. la post-maturation de la parole étrangère, les douleurs obstétricales de sa propre parole» (cité supra). En l'occurrence, la thèse de Benjamin, loin de sombrer dans la célébration de l'ineffable, rejoint plutôt la considération capitale de Wittgenstein au point 4.121 de son Tractarus: "Ce qui s'exprime dans la langue, nous ne pouvons par elle l'exprimer»30. On ne peut que faire encontre de ce hiatus abyssal où se dessine le passage du questionnement sur le statut ontologique de l'entité linguistique à celui de l'éthique qui nous

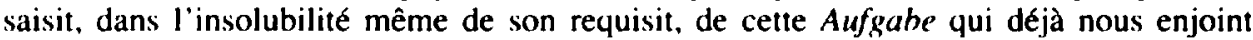
dans l'usage le plus prosaïque de notre langue maternelle en ce qu'elle ne laisse d'être fécondée par le rapport à l'étranger, tous métèques que nous sommes.

Notes

* Cet anticle a été rédigé dans le cadre d'une recherche subventionnée par le CRSH et dirigée par Alexis Nouss.

1. Willis Bamstone. The Poetics of Translation. Hissory. Theory. Practice. New Haven et Londres. Yale University Press. 198.3: 19-20.

2. George Steiner, After Babel: Aspects of Language and Translation, Oxford University Press, 1975: $73-74$

3. Voir G. 1. Bursill-Hall. Speculative Grammars of the Middle Ages: the Doctrine of "Partes Orationis", The Hague, Mouton, 1971 : Jan Pintorg. Medieval Semantics. Selected Studies an Medieval Logic and Grammar, Collected Studies Series 195, ed. par Sten Ebbesen, Londres, Variorum Reprints, 1984.

4. Antoine Amauld et Claude Lancelot, Grammaire générale é raisonnée. fac-similé de l'éd. de Paris, Le Petit, 1676 ( $3^{\mathrm{r}}$ ed. revuc et augmentéc de nouveau). Édition critique présentée par H. E. Brekle. StuttgartBad Cannstat, Frommann (Holeboog), "Grammatica universalis»1. 1966.

5. Antoine Amauld et Pierre Nicole, La logique' ou l'arl de penscr, fac-similé de l'éd. de Paris, Desprez, 1683 is éd. revue et augmentée de nouveau). Introduction par P. Roubinet. Publications de la Faculté des Lettres et Sciences humanes de I' Intversite de L.1lle, 1964.

6. Noam Chomsky, La lingussique cartésienne. Un chaputre de l'histoire de la pensée rationaliste, trad. par N. Delancei et D. Sperber, Paris, Seuil, $1969:$ 64-65. Pour une mise au point très précise sur l'existence d"une «linguistique cartésienne", voir André Joly, "La linquistique cartésienne : une erreur mémorable", La grommosire générole. Des modiste's aux ldéolongues. présenté par A. Joly et Jean Stéfanini. Publications de jUniversité de Lille. $1977: 165-199$.

7. Vour Julia M. Penn, Linguistic Relativity versus Innate Ideas: Origins of the Sapir-Whorf Hypothesis in Cierman Thoughr. The Hague, Mouton, “Janua Linguanum ", Series Minor 120. 1972.

8. Antoine Berman. L'epretu'e de l'éranger. Critique et traduction dans l'Allemagne romantique, Paris, Gallimard, 1984

9. W. Bamstone, $1993 ; 42$, = Jorge Luis Borges, "Translation", in Twenty-four Conversarions with Borges. Includeng a Selecrion of Prems: Intervien's by Roberto Alifano: 1981-1983. trad. par Nicomedes Arauz. Willis Barnstone et Noemi Escandell, New York, Grove Press, 1984.

10. Walter Benjamin, "Dic Aufgabe des Ühersetzers", in Illuminationen, Ausgewahlte Schriften I, Francfort, Suhrkamp. 1951 : 50) " "a tâche du traducteur", trad. par Maurice de Gandillac, in (Furres I: Mythe e't violence, Paris, Denoël, 1971 : 261-275, op. rit., 266.

11. Umberto Eco, Simurnique "t philosophie du langage", trad. par Myriem Bouzaher, Paris, PUF, I988, of. les chap. II et III.

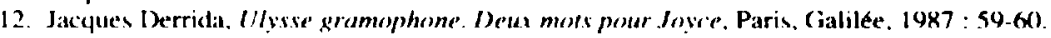

13. L. Lamy, “Du 'traduire" comme extradition». Discrurs social/Social Descrourse, vol. 5: 3-4. 199.3: 98. note 4.

14. J. Derrida. “Des tours de Babel”. Psyche. Inventions de lautre. Paris. Galilee, $1987: 203-235$; sur le rapport entre la théorie de l'Überséfang et la constellation sémantique qui se rattache à la notion romantique et idéaliste (Schelling) de la Bildung, regroupant entre autres les notions d'Urbild (idéal prototypique), d'Eimbildong (imagnation) el d'ln-tins.Bildung (a peu pres intraduisible : uni-formation?), voir aussi de J. Derrida, "Theologic de la Iraduction", Tex/, n" 4, 1985: 26-39.

15. W. Benjamin. "Thèses sur la philosophic de l'histoire", trad. par M. de Gandillac, (Eurres /I: Poésie ef revolution, Paris, Denceil, $1971: 278$

16. Jacyues Lacan, Érriss, Paris. Seuil, 1966: 94, où l'on peut lire : aL'assomption jubilatoire de son image spéculaire par l'être encore plongé dans l’impuissance motrice el la dépendance du nourrissage qu'est le petit homme a ce stade infons. nous paraîtra dès lors manifester en une situation exemplaire la matrice sym. 
bolique où le je se precipite en une forme primordiale. avant qu'il ne s'objective dans la dialectique de l'identification a l'autre el que le langage ne lui restitue dans l'universel sa fonction de sujet.m

17. Henri Meschonnic, “L'allégorie chez Walter Benjamin, une aventure juiven, Walter Benjamin et Paris. Colloque intemational. 27-29 juin 1983, ed. par Heinz Wismann, Paris, Cerf, 1986 : 709.

18. Giorgio Agamben, La communauté qui vient. Théorie de la singularite quelconque, Irad. par Marilene Raiola, Paris, Seuil, $1990: 95$ et 113.

19. George Steiner, Les Antigones, trad. par Philippe Blanchard, Paris, Gallimard, 1986.

20. Voir Roben M. Adams. Proteus. His Lies, His Truth: Discussions of Literary Translation. New York. W. W. Norton. $1973: 6$.

21. Voir Gregory Bateson, Vers une écologie de l'esprit, en deux tomes, trad. par Ferial Drosso, Laurencine Lot, avec le concours d'Eugène Simion et Christian Cler, Paris, Seuil, 1977 el 1980 ; consulter l'index dans le tome second à la rubrique adouble contrainte». Il est tout de méme étonnant que Derrida et ses épigones. qui usent abondamment de la notion de double bind, ne fassent jamais référence aux travaux de Bateson.

22. George Lakoff. Women. Fire. and Dangerous Things. What Categories Reveal ahout the Mind. Chicago et Londres. The University of Chicago Press. 1987.

23. J'emprunte cette notion à Daniel C. Dennett, telle qu'il l'a développée dans son dernier ouvrage Consciousness Explained. Boston, Little, Brown and Co., 1991.

24. Éliane Amado Lévy-Valensi, La naturé de la pensée inconsciente, Paris, Éditions Universitaires/JeanPierre Delarge. $1978: 72$.

25. J. Derrida a consacré une importante étude à cette question, en relation précisement avec le probleme de la traduction, "Freud et la scène de l'ecriture", L'écrifure et la différence. Paris. Seuil, $1967: 293-340$.

26. Yvon Gauthier, De la logique interne. Paris. Vrin. 1991: 13.

27. Ces deux passages sont cités et traduits in G. Agamben, "Langue et histoire. Catégories historiques et catégories linguistiques dans la pensee de Benjamin». Walter Benjamin et Paris, op. cit., 796-797.

28. Voir Jürgen Habermas, Theoric de lagir communicarionnel, deux tomes, trad. par Jean-Marc Ferry, Paris. Fayard. 1987: Karl-Otto Apel. Sur le prohlème d'une fondation rationnelle de l'sihique d l'age de la science. L'a prioriv de la communauté communicationnelle ef les fondements de l'éthique, trad. par R. Lellouche et I. Mittmann. Presses Universitaires de Lille, 1987; de Apel, voir aussi "La fondation pragmatico-transcendantale de lentente communicationnelle illimitee", trad. par Denis Dumas, La communauté en paroles. Communauté, consensus, ruptures, ed. sous la direction de Herman Parret, Lieze. Pierre Mardaga, $1991: 15-33$.

29. Ludwig Wiltgenstein, Tractatus logico-philosophicus, traduction, prtambule et notes de Gilles-Gaston Granger, Paris, Gallimard. $1993: 58$.

30. W. V. O. Quine, Word and Ohject. Cambridge. MA. MIT Press, 1960, chap. 2. 